\title{
MACROFAUNA DO SOLO NO PARQUE MUNICIPAL DE MACEIÓ, ALAGOAS
}

\author{
Renato Wilian Santos de Lima \\ Universidade Federal de Alagoas (UFAL) \\ Instituto de Geografia, Desenvolvimento e Meio Ambiente, Maceió, AL, Brasil \\ Graduando em Geografia (Licenciatura) \\ renato6609@gmail.com
}

Carlos Augusto Rocha da Silva Universidade Federal de Alagoas (UFAL), Instituto de Geografia Desenvolvimento e Meio Ambiente, Maceió, AL, Brasil Graduando em Geografia (Bacharelado) carlosaugusto.silva777@hotmail.com

Delane dos Santos Dias Universidade Federal de Alagoas (UFAL) Instituto de Geografia, Desenvolvimento e Meio Ambiente, Maceió, AL, Brasil Mestranda em Geografia, do Programa de Pós Graduação em Geografia delane.sd@hotmail.com

Élida Monique da Costa Santos Universidade Federal de Alagoas (UFAL) Instituto de Ciências Biológicas e da Saúde

Doutoranda do Programa de Pós-Graduação em Diversidade Biológica e Conservação nos Trópicos elidamoniquecs@outlook.com

Danúbia Lins Gomes Universidade Federal de Alagoas (UFAL) Instituto de Ciências Biológicas e da Saúde Doutoranda do Programa de Pós-Graduação em Diversidade Biológica e Conservação nos Trópicos dlinsgomes@yahoo.com.br

Kallianna Dantas Araujo Universidade Federal de Alagoas (UFAL) Instituto de Geografia, Desenvolvimento e Meio Ambiente, Maceió, AL, Brasil Docente do Programa de Pós Graduação em Geografia kallianna.araujo@igdema.ufal.br

\begin{abstract}
RESUMO
Objetivou-se avaliar a macrofauna do solo em Unidade de Conservação, em Maceió, Alagoas, nos ambientes conservado, transição e alterado, de ago/2018 a jun/2019. Utilizouse armadilhas Provid que permaneceram no campo por 96 horas. Os organismos ( $>2 \mathrm{~mm}$ de comprimento) foram identificados a nível de ordem. Foi quantificada a abundância e aplicados os índices ecológicos de Shannon $(\mathrm{H})$ e Pielou (e). Quantificou-se o conteúdo de água do solo (CAS), e temperatura do solo (TS) na profundidade $0,10 \mathrm{~m}$, e precipitação pluvial (PP). Os dados foram submetidos à análise de variância, tendo suas médias comparadas pelo teste de Tukey a $5 \%$ de probabilidade. O ambiente conservado apresenta maior riqueza e abundância, em razão da variedade de alimento e abrigo na serapilheira, aliado aos fatores abióticos CAS e TS; Hymenoptera, adaptado às condições antrópicas, é dominante nos ambientes, comprovado pelos baixos valores dos índices $(\mathrm{H})$ e $(e) ; \mathrm{Na}$ área conservada Hymenoptera não foi discrepante em relação aos demais grupos, refletindo o equilíbrio da área. A variável TS influenciou a abundância, o CAS apresentou influência na riqueza e a PP influenciou ambas as variáveis.
\end{abstract}

Palavras-chave: Mata Atlântica. Organismos Edáficos. Qualidade do Solo. Índices Ecológicos.

\section{SOIL MACROFAUNA AT MACEIÓ MUNICIPAL PARK, ALAGOAS}

\section{ABSTRACT}

The objective of the present study was to evaluate the soil macrofauna in a Conservation Unit, in Maceió, Alagoas, in the conserved, transition environments and changed in Aug/2018 to Jun 2019. Provid traps were used and remained in the field for 96 hours. The organisms (> 2 
$\mathrm{mm}$ in length) were identified at the order of the level. The abundance was quantified and the Shannon $(\mathrm{H})$ and Pielou $(e)$ ecological indexes were applied. Soil water content (SWC) and soil temperature (ST) were quantified at a depth of $0.10 \mathrm{~m}$ and rainfall $(\mathrm{R})$. The data were submitted to analysis of variance, with their averages compared by the Tukey test at $5 \%$ probability. The conserved environment presents greater wealth and abundance, due to the variety of food and shelter in the litter, combined with the abiotic factors SWC and ST; Hymenoptera, adapted to anthropic conditions, is dominant in environments, proven by the low values of the $(\mathrm{H})$ and $(e)$ indexes; In the conserved area, Hymenoptera was not inconsistent in relation to the other groups, reflecting the balance of the area. The ST variable influenced the abundance, the SWC had an influence on wealth and the R influenced both variables.

Keywords: Atlantic Forest. Edaphic Organisms. Soil Quality. Ecological Indexes.

\section{INTRODUÇÃO}

A macrofauna edáfica é composta por organismos com comprimento superior a $2 \mathrm{~mm}$ (SWIFT et al., 1979), representada por mais de 20 grupos taxonômicos, destacando-se Hymenoptera, Araneae, Coleoptera, Diplopoda, Isoptera, Chilopoda, dentre outros (AQUINO et al., 2008; MELO et al., 2009; BARETTA et al., 2011).

A maior parte desses invertebrados está presente em até $0,30 \mathrm{~m}$ da superfície (RUIZ et al., 2008) e são capazes de se movimentarem horizontalmente e verticalmente, possibilitando a distribuição de matéria orgânica e minerais através das galerias, considerados agentes ecológicos e funcionais na estruturação dos solos (PATUCCl et al., 2015).

Os organismos da macrofauna edáfica são capazes de fornecer informações sobre o equilíbrio dos ecossistemas, são responsáveis pela decomposição da matéria orgânica, formação e manutenção do solo, dispersão de sementes, produção de oxigênio, fixação de carbono, controle biológico, considerados indicadores da qualidade do solo (PATUCCI et al., 2018; POMPEO et al., 2016; FILSER et al., 2016; SOUZA et al., 2015; LAVELLE et al,. 2006), além de indicadores de recuperação e restauração de áreas florestais perturbadas e degradadas (POMPEO et al., 2016; MACHADO et al., 2015).

Fatores de ordem natural regulam a estrutura e abundância da fauna edáfica em ecossistemas tropicais, como o microclima (MARQUES e DEL-CLARO, 2010), especialmente, a precipitação pluvial e temperatura, que podem influenciar no padrão de distribuição e diversidade de muitos grupos biológicos (ALMEIDA et al., 2015).

A literatura tem demonstrado que ambientes conservados favorecem a riqueza faunística, conforme apontam Garcia e Catanozi (2011) ao estudarem uma área de mata atlântica conservada, e esta apresentou maior registro de diversidade, densidade de organismos e riqueza de grupos em comparação ao ambiente com reflorestamento de Pinus sp.

O bioma mata atlântica apresenta alta diversidade de espécies, elevada taxa de endemismo e por apresentarem elevados níveis de ameaça de extinção de espécies da fauna, flora e da introdução de espécies exóticas, foi apontado como um dos hotspots mundiais nos estudos de Myers et al. (2000) e Mittermeier et al. (2005).

O Parque Municipal de Maceió, em Alagoas, localizado no bairro Bebedouro, possui uma área de 82,4 ha é considerado uma Unidade de Conservação (LIMA, 2009), possui uma área representativa da Mata Atlântica que, de acordo com o SNUC, está enquadrada na categoria das unidades de proteção integral, tendo os conceitos de um parque nacional, porém, pelo fato de ser gerido pelo órgão ambiental da esfera municipal, a Secretaria Municipal de Desenvolvimento Sustentável - SEMDS, sua nomenclatura se torna Parque Municipal de acordo com a lei № 2514/78 (SILVA, 2017). Conta com várias espécies 
de árvores da Mata Atlântica, com destaque para as espécies Pterodon emarginatus (sucupira), Cecropia glaziouvi (embaúba), Paubrasilia echinata (pau-brasil), Tabebuia caraiba Mart. (craibeira), Handroanthus avellanedae (ipê-roxo), Handroanthus ochraceus (ipê-amarelo), Handroanthus avellanedae (ipê-rosa), Handroanthus roseoalba (ipê-branco), Eschweilera ovata (embiriba), Caesalpinia ferrea (pau-ferro), Inga (ingazeiro), dentre outras (PIMENTEL, 2010) e é aberto à visitação ao público, dispondo de cinco trilhas ecológicas, onde é possível observar trechos de mata conservados, em transição e alterados e o entorno do Parque é circundado pela comunidade local, tornando-o vulnerável a ação antrópica (SILVA, 2017; SILVA, 2011). Desse modo, esta pesquisa apresenta um caráter pioneiro pelo levantamento da fauna do solo ter sido realizado em ambientes com diferentes tipos de cobertura vegetal em um Parque Municipal urbano.

Procurou-se investigar qual a composição da macrofauna do solo nos ambientes conservado, em transição e alterado, na Unidade de Conservação, em Maceió, Alagoas? Para responder este questionamento foi elaborada a hipótese: a composição da macrofauna do solo é maior no ambiente conservado, influenciada pelas condições microclimáticas locais, com picos de aumento em condição favorável do substrato, havendo decréscimo da riqueza, abundância, diversidade e equabilidade quando há uma situação desfavorável.

Nesse sentido, o objetivo do trabalho foi avaliar a macrofauna do solo em Unidade de Conservação, em Maceió, Alagoas. Para tanto foram comparados três ambientes distintos: conservado, transição e alterado ao longo de um ano com leituras bimestrais.

\section{METODOLOGIA}

\section{Local da Pesquisa}

A área de estudo (Figura 1) está localizada em três ambientes: conservado (Figura 2A), em transição (Figura 2B) e alterado (Figura 2C), no Parque Municipal que é uma Unidade de Conservação, localizado nas coordenadas geográficas $9^{\circ} 36^{\prime} 47.4^{\prime \prime} \mathrm{S}$ e $35^{\circ} 45^{\prime} 36.9 \mathrm{~W}$, com área de 82,4 ha, localizado no bairro Bebedouro, em Maceíó, Alagoas, inserido na Mesorregião Geográfica do Leste Alagoano e Microrregião de Maceió.

Possui clima As' - Tropical quente com chuvas de outono/inverno, segundo a classificação de Köppen (PEEL et al., 2007), com precipitação de $1.867,4 \mathrm{~mm} / \mathrm{ano}$, temperatura do ar de $25,1^{\circ} \mathrm{C} /$ ano e umidade relativa de 78,5\%/ano (INMET, 2018). Apresenta topografia irregular, variações de altitude, abrangendo vales e encostas que atingem $75 \mathrm{~m}$ e bioma do tipo mata atlântica remanescente (Floresta Ombrófila Aberta) (SILVA, 2011; LIMA, 2009). Os solos são do tipo Latossolos Vermelho Amarelo Distrófico, Argissolo Vermelho Amarelo e Argissolo Vermelho Amarelo latossólico e Solos aluviais no fundo do vale (EMBRAPA, 2012).

Quanto à hidrografia local, o Parque Municipal está inserido na Bacia do Riacho do Silva, que possui nascentes dentro da própria unidade (SILVA, 2017). A unidade está inserida no compartimento litoestrutural da Província Costeira, compreendida por um pacote sedimentar representado pela Bacia Sedimentar de Alagoas, onde ocorrem depósitos de idade cenozóica (quaternários e terciários) e Paleomesozóico (SILVA, 2017; SILVA, 2011). As estruturas geomorfológicas encontradas são a Planície Litorânea dos Piemontes Inumados (Tabuleiros Costeiros), com a Planície sendo a menor em expressão espacial, tendo origem quaternária e predominando a acumulação flúvio-lagunar. Os Tabuleiros Costeiros possui uma superfície composta basicamente por terrenos plio-pleistocênicos (Planalto Sedimentar Costeiro), sendo que no Parque Municipal de Maceió as encostas são estuarinas lagunares e interflúvios tabuliformes dissecados (SILVA, 2017; SILVA, 2011). 
Renato Willian Santos de Lima Carlos Rocha Augusto da Silva Delane dos Santos Dias Élida Monique da Costa Santos Danúbia Lins Gomes

Figura 1 - Localização dos três ambientes pesquisados (Conservado, em Transição e Alterado), no Parque Municipal, Maceió, Alagoas.

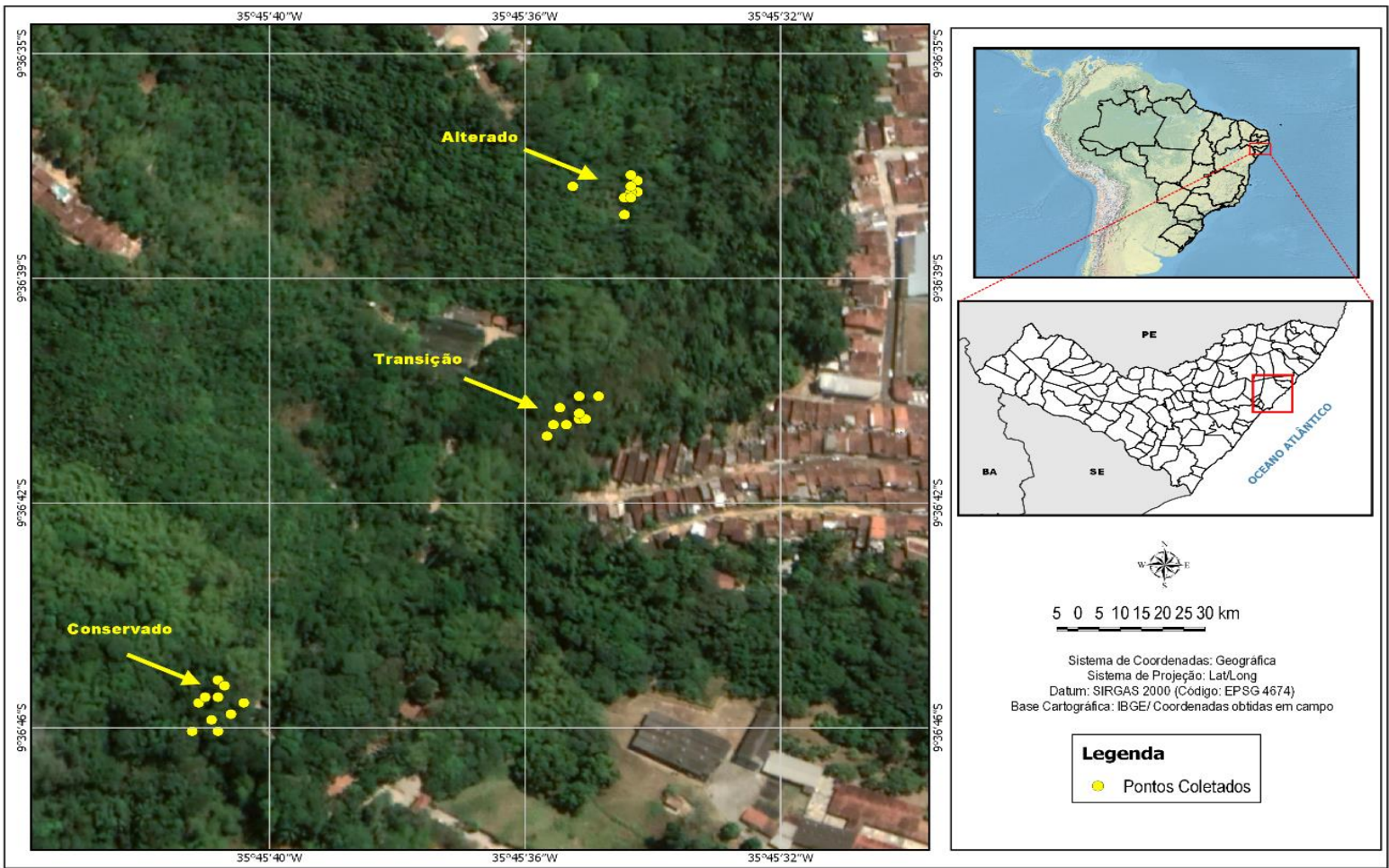

Elaborado por - Lionaldo dos Santos (2019).

Figura 2 - Ambientes avaliados: conservado (A), transição (B) e alterado (C).
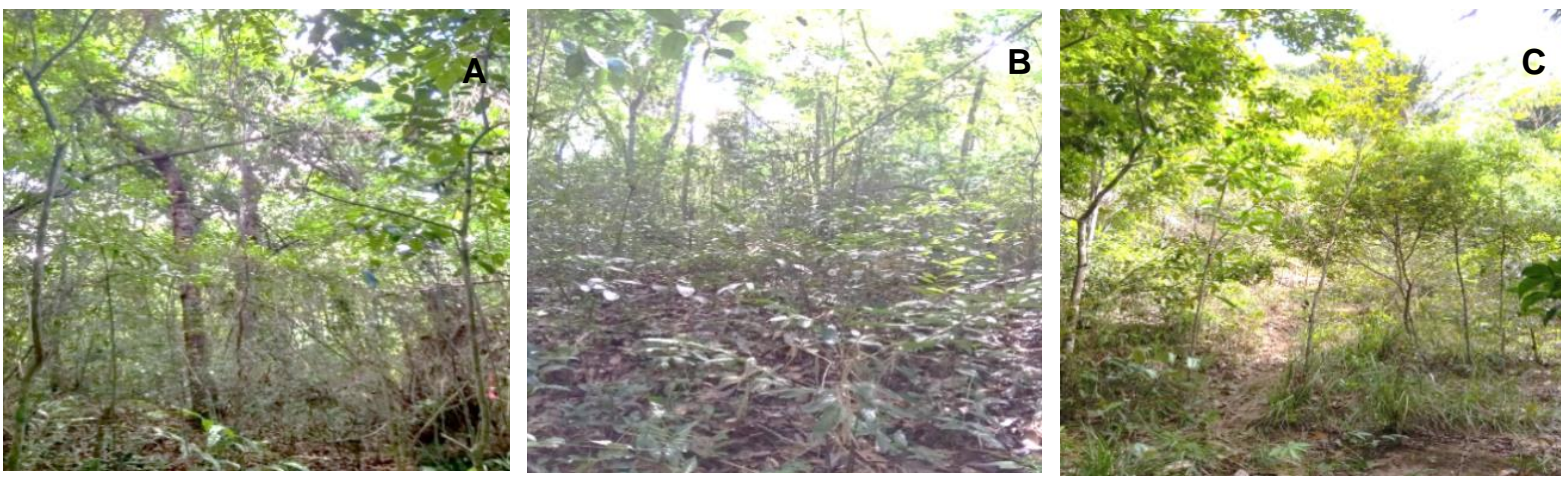

Fonte - Autores (2019).

\section{Quantificação da macrofauna do solo}

A quantificação da macrofauna foi realizada nos três ambientes (conservado, transição e alterado) bimestralmente em 10 pontos (ago/out/dez de 2018 e fev/abr/jun de 2019), utilizando armadilhas Provid, com quatro orifícios de dimensões $2 \times 2 \mathrm{~cm}$ (ARAUJO, 2010), constituídas de garrafa PET $2 \mathrm{~L}$, contendo $200 \mathrm{~mL}$ de solução de detergente, na concentração de $5 \%$ e 12 gotas de Formol P.A. (Formaldeído) que permaneceram no campo por 96 horas, enterradas com os orifícios ao nível da superfície do solo (Figura 3A). As armadilhas foram mantidas no mesmo local ao longo do tempo, sendo 10 repetições em cada ambiente. 
Após recolhidas, o material coletado foi lavado em água corrente, com o auxílio de uma peneira de 0,25 $\mathrm{mm}$ (Figura 3B) e por meio de lupa e pinças foi feita a contagem dos organismos (> $2 \mathrm{~mm}$ de comprimento) e estes foram armazenados em solução de álcool a 70\% (SWIFT et al., 1979) e identificados a nível de ordem dos grandes grupos taxonômicos utilizando a chave de identificação de Triplehorn e Jonnson (2011) (Figura 3C).

Figura 3 - Instalação das armadilhas (A), lavagem do material (B), contagem e identificação dos organismos $(\mathrm{C})$.
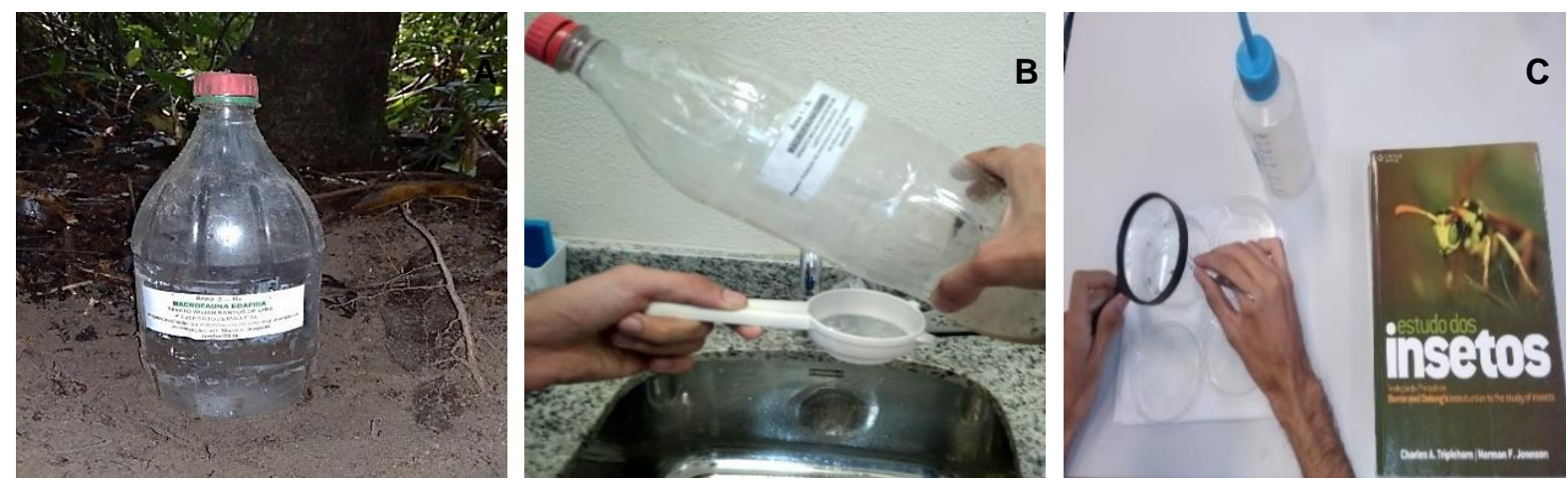

Fonte - Autores (2019).

A macrofauna foi avaliada de forma quantitativa pela abundância de espécimes e qualitativa mediante diversidade. A diversidade foi calculada pelo Índice de Diversidade de Shannon $(\mathrm{H})$, definido por: $\mathrm{H}=-$ $\Sigma$ pi.logpi, em que: $\mathrm{pi}=\mathrm{ni} / \mathrm{N}$; $\mathrm{ni}=$ densidade de cada grupo, $\mathrm{N}=\Sigma$ da densidade de todos os grupos e a equabilidade pelo Índice de Pielou (e) definido por: e=H/log S, em que: $\mathrm{H}=$ índice de Shannon; $\mathrm{S}=$ Número de espécies ou grupos.

O índice $(\mathrm{H})$ varia de 0 a 5 , indicando que o declínio de seus valores é o resultado de uma maior dominância de grupos em detrimento de outros (BEGON et al., 1996). O Índice de Equabilidade de Pielou (e) varia de 0 (uniformidade mínima) a 1 (uniformidade máxima), permitindo representar a uniformidade da distribuição dos indivíduos entre as espécies existentes (PIELOU, 1977).

Foram realizadas determinações do Conteúdo de Água do Solo (CAS) bimestralmente (ago/out/dez de 2018 e fev/abr/jun de 2019) nos mesmos pontos de coleta da macrofauna, na profundidade $0,10 \mathrm{~m}$. As amostras de solo foram pesadas em balança analítica para obter o peso úmido e, em seguida, foram levadas para estufa sem circulação de ar para secagem durante 24 horas e novamente pesadas para obtenção do peso seco, com base na metodologia de Tedesco et al. (1995), pela equação: CAS\% = $((\mathrm{Pu}-\mathrm{Ps}) / \mathrm{Ps}) \times 100$, em que: $\mathrm{CAS}=$ Conteúdo de água do solo; $\mathrm{Pu}=$ Peso do solo úmido (g); Ps = Peso do solo seco (g).

Em razão da maior atividade dos organismos edáficos na camada superficial do solo, foram realizadas medidas de temperatura do solo na profundidade $0,10 \mathrm{~m}$, com termômetro digital espeto nos três ambientes. Também foram obtidos dados de precipitação pluvial da estação meteorológica do LABESA/IGDEMA/UFAL e do INMET (Instituto Nacional de Meteorologia).

\section{ANÁLISE ESTATÍSTICA}

Os dados da macrofauna edáfica foram analisados pela estatística descritiva e submetidos à análise de variância, tendo suas médias comparadas pelo teste de Tukey a $5 \%$ de probabilidade, visando verificar se houve diferença entre os dados nas áreas.

$\begin{array}{lllll}\text { Caminhos de Geografia } \quad \text { Uberlândia-MG } & \text { v. 22, n. } 81 & \text { jun./2021 } & \text { p. 292-307 Página } 296\end{array}$


Estimativas de correlação de Pearson foram realizadas entre os dados de abundância e riqueza da macrofauna edáfica, com as variáveis ambientais (conteúdo de água do solo, temperatura do solo e precipitação pluvial). A significância foi verificada usando o teste t de Student a $5 \%$ de probabilidade. O intuito desta análise foi testar o grau variáveis dependentes ou respostas (abundância e riqueza da macrofauna) em relação às independentes ou explicativas (precipitação pluvial, temperatura do solo e conteúdo de água do solo).

A interpretação dos resultados da estimativa de correlação foi baseada na significância e classificação proposta por Dancey e Reidy (2006) na qual aponta uma correlação: fraca $(\rho \leq 0,399)$, moderada ( $\rho \geq$ $0,400 \leq 0,700)$ e forte $(\rho \geq 0,701)$. Ambos os testes foram realizados no software $R$ versão 3.4 .4 ( $R$ CORE TEAM, 2019).

\section{RESULTADOS E DISCUSSÃO}

\section{QUANTIFICAÇÃO DA MACROFAUNA EDÁFICA DO PARQUE MUNICIPAL DE MACEIÓ, ALAGOAS}

Nas coletas de agosto, outubro e dezembro de 2018 e fevereiro, abril e junho de 2019 foram capturados 7.781 indivíduos, distribuídos entre 19 grupos taxonômicos com 3.828 indivíduos no ambiente conservado, 1.977 em transição e 1.976 no ambiente alterado, distribuídos em 17, 17 e 15 grupos, respectivamente (Figura 4).

Figura 4 - Abundância e riqueza da macrofauna edáfica.

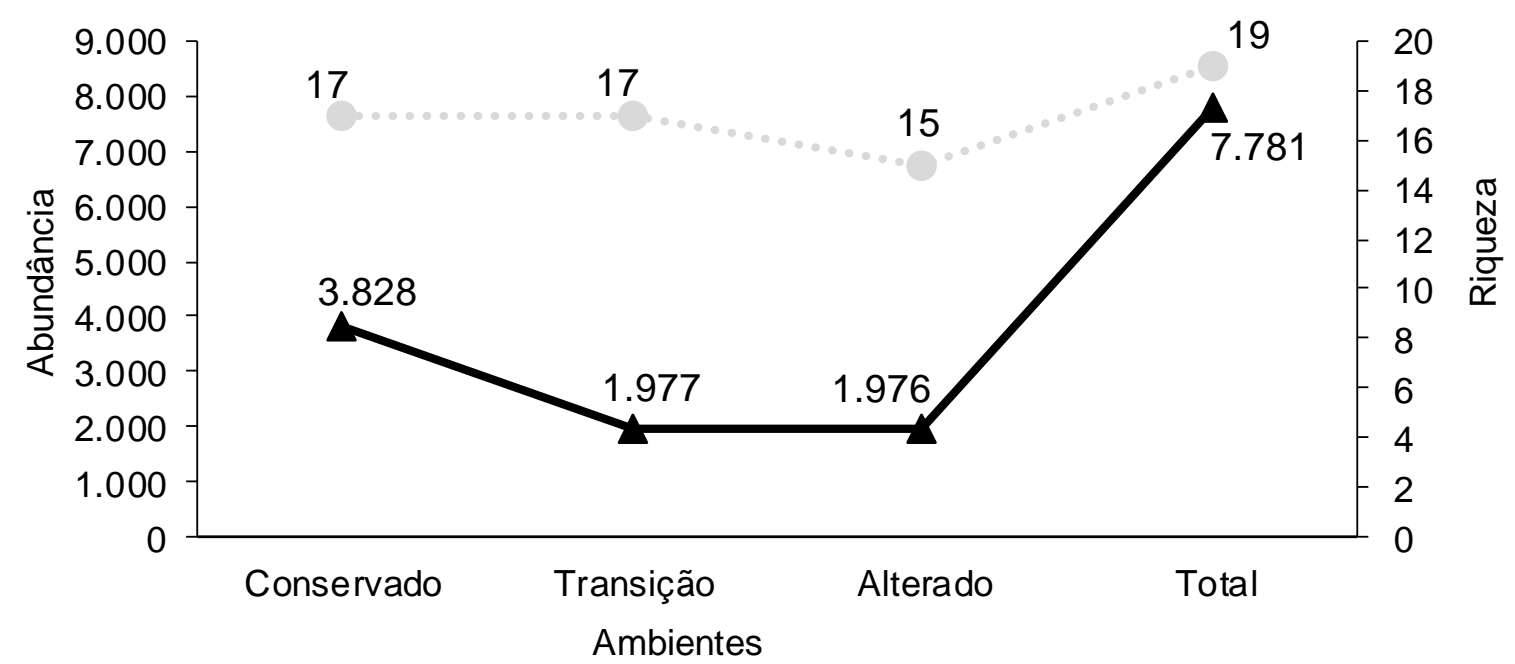

- Abundância

Riqueza

Fonte - Autores (2019).

Independente dos ambientes houve maior abundância do grupo Hymenoptera com valores correspondentes a $71,42 \%$ no ambiente em transição, $70,34 \%$ no alterado e $33,86 \%$ no ambiente conservado (Figuras 5A a 5C). O elevado percentual do grupo Hymenoptera nos ambientes em transição (onde há descarte de lixo irregular pela população local) (Figuras 6A a 6C) e alterado (onde há presença de espécies exóticas ao bioma) ocorreu independente das intervenções antrópicas as quais os ambientes foram submetidos. Esse resultado mostra que este grupo é resistente as condições severas do ambiente. Tem capacidade de ocupar diversos locais (SILVA et al., 2014), sendo capazes 
de colonizar ambientes terrestres que não oferecem muitos recursos para o desenvolvimento (BARETTA et al., 2011). Apresentando-se como indicadores de perturbação do meio edáfico devido sua habilidade para sobreviver em solos com distúrbios do meio, o que se evidencia a predominância, abundância e resistência em sistemas que possuem algum nível de intervenção antrópica (CABRERA, 2012).

Figura 5 - Percentual da macrofauna edáfica nos ambientes conservado (A), em transição (B) e alterado (C).

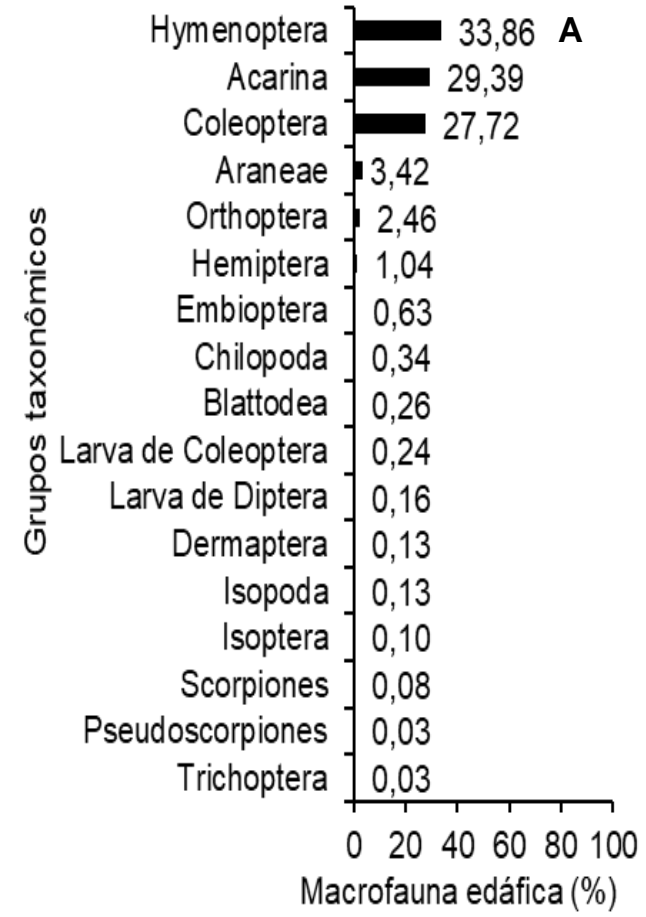

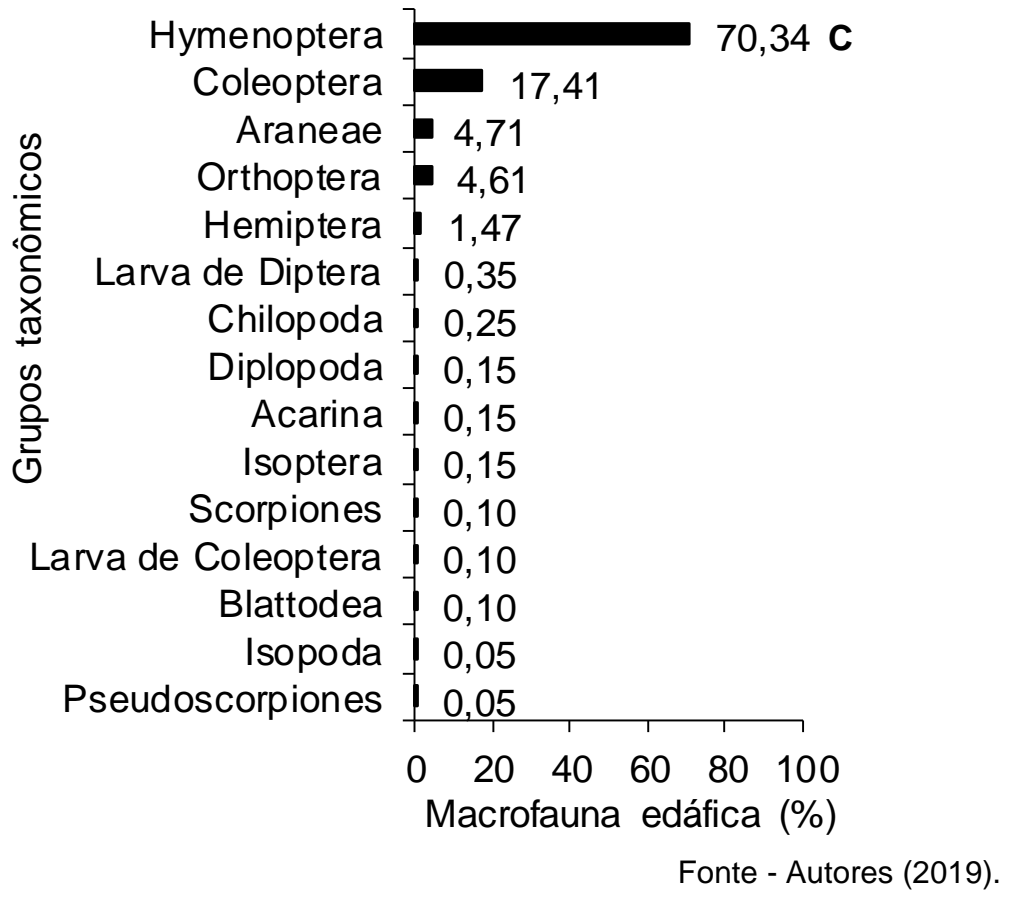

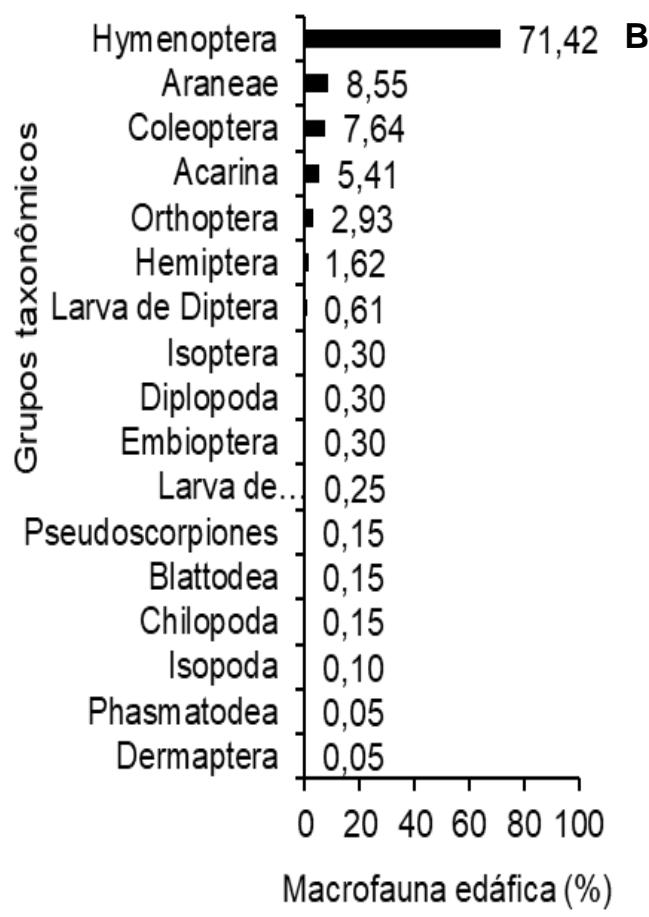


Figura 6 - Descarte irregular de lixo no entorno da área de transição $(A, B, C)$ pela população residente que circunda o Parque Municipal de Maceió
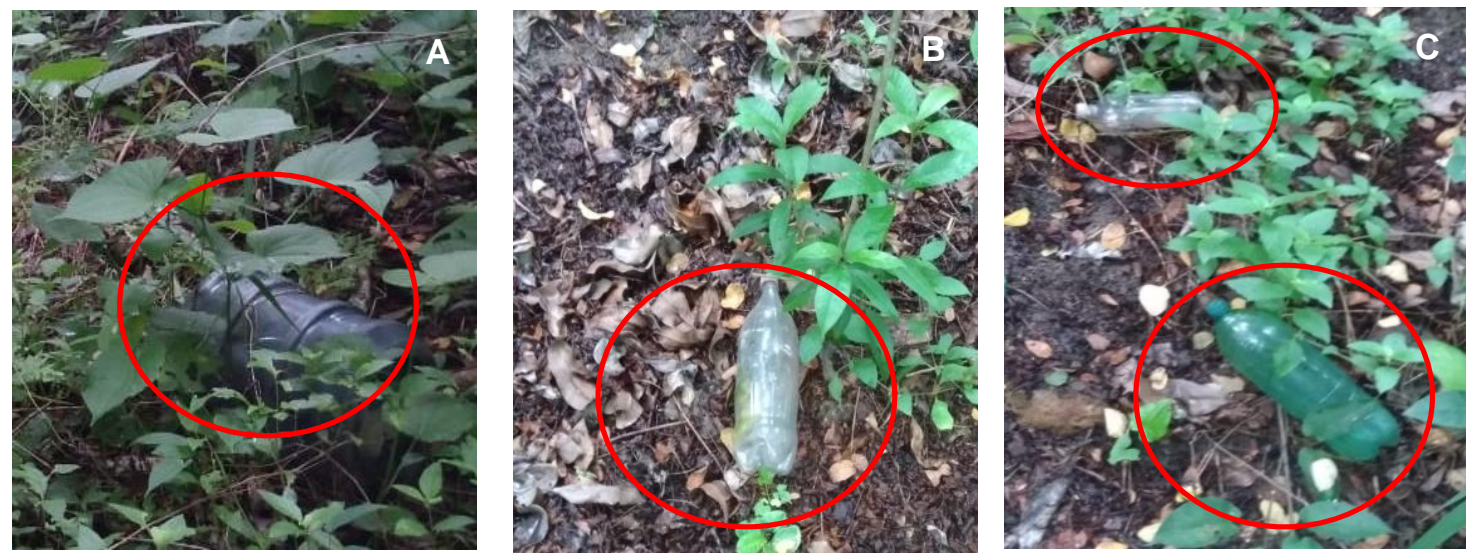

Fonte - Autores (2019).

$\mathrm{Na}$ área conservada destacaram-se três grupos taxonômicos Hymenoptera $(33,86 \%)$, seguido de Acarina $(29,34 \%)$ e Coleoptera $(27,72 \%)$ que somados constituíram $90,97 \%$ dos organismos (Figuras $5 \mathrm{~A}$ a $5 \mathrm{C}$ ). $\mathrm{O}$ grupo Hymenoptera devido ao seu hábito de vida colonial, tendem a ser amostrados com elevados números de indivíduos. Mas pode-se observar que na área conservada o percentual de sua abundância não foi tão discrepante quando comparado aos outros grupos que também apresentam características de grupos abundantes (o que não é possível ser observado na área de transição e no ambiente alterado). Isso mostra como a área conservada está em equilíbrio, ofertando condições favoráveis para diversos grupos.

Resultados similares foram obtidos em estudo realizado por Pech et al. (2016), em ambiente de regeneração na mata atlântica do município Curitibanos, Santa Catarina, onde registraram maior abundância do grupo Hymenoptera, seguido por Coleoptera e Araneae.

Em área de fragmento florestal de mata atlântica no município Macaíba, Rio Grande do Norte e em fragmento remanescente de mata Atlântica em Areia, Paraíba, respectivamente Silva et al. (2014) e Alves et al. (2014), detectaram Hymenoptera com registro acima de $30 \%$, maior valor em relação às demais ordens taxonômicas.

Os menores valores dos índices de diversidade e uniformidade foram para o grupo Hymenoptera nas áreas conservada $(H=0,47 ; e=0,13)$, transição $(H=0,15 ; e=0,04)$ e alterada $(H=0,15 ; e=0,05)$ (Tabela 1), pois são os mais dominantes nas áreas, por apresentarem maior adaptabilidade aos ambientes, mesmo os que oferecem poucos recursos alimentares e abrigo (MELO et al., 2009).

Tabela 1 - Índices de diversidade de Shannon $(\mathrm{H})$ e Índice de uniformidade de Pielou (e) dos grupos taxonômicos registrados nos ambientes conservado, em transição e alterado.

\begin{tabular}{lcccccc}
\hline \multirow{2}{*}{ Grupos taxonômicos } & \multicolumn{9}{c}{ Ambientes } \\
\cline { 2 - 8 } & \multicolumn{2}{c}{ Conservado } & \multicolumn{2}{c}{ Transição } & \multicolumn{2}{c}{ Alterado } \\
\cline { 2 - 8 } & $\mathrm{H}$ & $e$ & $\mathrm{H}$ & $e$ & $\mathrm{H}$ & $e$ \\
\hline Hymenoptera & 0,47 & 0,13 & 0,15 & 0,04 & 0,15 & 0,05 \\
Acarina & 0,53 & 0,15 & 1,27 & 0,38 & 2,82 & 0,86 \\
Coleoptera & 0,56 & 0,16 & 1,12 & 0,34 & 0,76 & 0,23 \\
Araneae & 1,47 & 0,41 & 1,07 & 0,32 & 1,33 & 0,40 \\
Orthoptera & 1,61 & 0,45 & 1,53 & 0,46 & 1,34 & 0,41
\end{tabular}




\begin{tabular}{lcccccc} 
Hemiptera & 1,99 & 0,55 & 1,79 & 0,54 & 1,83 & 0,56 \\
Embioptera & 2,20 & 0,61 & 2,52 & 0,76 & - & - \\
Chilopoda & 2,47 & 0,69 & 2,82 & 0,86 & 2,60 & 0,79 \\
Blattodea & 2,58 & 0,72 & 2,82 & 0,86 & 2,99 & 0,91 \\
Larva de Coleoptera & 2,63 & 0,73 & 2,60 & 0,79 & 2,99 & 0,91 \\
Larva de Diptera & 2,80 & 0,78 & 2,22 & 0,67 & 2,45 & 0,74 \\
Dermaptera & 2,88 & 0,80 & 3,30 & 1 & - & - \\
Isopoda & 2,88 & 0,80 & 2,99 & 0,91 & 3,30 & 1 \\
Isoptera & 2,98 & 0,83 & 2,52 & 0,76 & 2,82 & 0,86 \\
Scorpiones & 3,11 & 0,87 & - & - & 2,99 & 0,91 \\
Pseudoscorpiones & 3,58 & 1 & 2,82 & 0,86 & 3,30 & 1 \\
Trichoptera & 3,58 & 1 & - & - & - & - \\
Phasmatodea & - & - & 3,30 & 1 & - & - \\
Diplopoda & - & - & 2,52 & 0,76 & 2,82 & 0,86 \\
\hline
\end{tabular}

Fonte - Autores (2019).

\section{DETERMINAÇÃO DO CONTEÚDO DE ÁGUA DO SOLO (CAS) E PRECIPITAÇÃO PLUVIAL (MM)}

No ambiente conservado constatou-se maior riqueza e abundância da macrofauna edáfica, atribuído às condições favoráveis como alimento e abrigo encontrado no solo e na espessa camada de serapilheira, além de proteção do solo pela copa das árvores (Figura 4).

Conforme afirma Penereiro (1999), em ambiente de mata há um ciclo completo de queda de folhas e galhos, além da boa condição do microclima. E isso acaba influenciando o desenvolvimento dos organismos no local. Essa camada pode regular a entrada de água das chuvas e de nutrientes para o interior do solo (MATEUS et al., 2013) pelo processo de lixiviação, sendo a serapilheira considerada um compartimento do ecossistema com importância hidroecológica (CIANCIARUSO et al., 2006), já que desempenha um papel fundamental na regulação dos processos hidrológicos superficiais (SATO, 2008). As camadas de serapilheira com maior grau de decomposição apresentam maior superfície específica e, dessa maneira, seu potencial de retenção hídrica é maior quando comparado ao de camadas mais superficiais e não decompostas (VALLEJO, 1982). E através dos diferentes estágios de decomposição, a camada de serapilheira é responsável pelo armazenamento de água no solo, bem como pelo aumento das taxas de infiltração e condicionamento dos fluxos superficiais, além de melhorar a administração de água ao solo, via fluxos subsuperficiais, disponibilizando água e meios aos indivíduos que irão (re)construir os ecossistemas, dando celeridade aos processos de restauração (OLIVEIRA, 1987). Em Floresta Tropical Úmida, por exemplo, a serrapilheira é bem heterogênea na sua composição e apresenta espessura variável, podendo incluir o horizonte $\mathrm{O}_{1}$, constituído por material recém caído (folhas, galhos, material reprodutivo), e a camada inferior, $\mathrm{O}_{2}$, que já apresenta sinais de alteração física e bioquímica. Nesta camada inferior a desagregação e decomposição microbiana dos materiais favorecem o aumento da superfície específica, acarretando numa maior capacidade de retenção hídrica desta camada em relação a O ${ }_{1}$ (VALLEJO, 1982).

Assim, a camada de serapilheira fornece maior variedade de alimentos para os organismos, favorecendo maior heterogeneidade de grupos. Confirmando o que afirmou Merlim (2005), que os

$\begin{array}{lllll}\text { Caminhos de Geografia } \quad \text { Uberlândia-MG } & \text { v. 22, n. } 81 & \text { jun./2021 } & \text { p. 292-307 Página } 300\end{array}$


organismos do solo são influenciados pela quantidade e, principalmente, pela qualidade do material vegetal que aporta ao solo.

O conteúdo de água do solo registrado no ambiente alterado $(9,81 \%)$ foi superior ao ambiente em transição $(8,62 \%)$ e conservado $(7,86 \%)$ (Figura 7$)$, decorrente da menor camada de serapilheira acumulada, quando comparado aos outros dois ambientes (em transição e conservado), uma vez que a manutenção da umidade no solo tem relação direta com a serapilheira que é um compartimento de estocagem de água, que permite a manutenção de certo grau de umidade no solo e, ao reter a água da chuva, vai liberando gradualmente para dentro do solo (MIRANDA, 1992), cuja média nos seis meses de coleta oscilou entre $5 \mathrm{~mm}$ (outubro/2018) e 154,2 mm (abril/2019) (Figura 8). Assim, na área de mata, logo após um evento de chuva, a água fica retida no compartimento serapilheira e lentamente vai sendo infiltrado no solo e isto está associado ao material predominante na serapilheira como espécies com maior aporte, textura das folhas, tipo de material (folhas, galhos, frutos, estrutura reprodutiva, miscelânea) e grau de decomposição, responsável por aumentar a capacidade de absorção e armazenamento de umidade. Sendo necessário a continuação de pesquisas nas áreas experimentais que foque na quantificação da capacidade de retenção hídrica da serapilheira acumulada sobre o solo. Pois a serapilheira fornece proteção ao solo, interceptando a água das chuvas (ARCOVA et al., 2003; MORAES, 2002), evita sua selagem, minimiza as taxas de evaporação, velocidade da enxurrada e, consequentemente, reduz o processo erosivo (VALLEJO, 1982). Além disso, a espessura da serapilheira modifica a qualidade espectral da luz e a temperatura que ultrapassa o estoque de matéria morta sobre o solo (FACELLI e PICKETT, 1991).

Já a temperatura do solo registrada do ambiente conservado foi a mais elevada, correspondendo a $25,74^{\circ} \mathrm{C}$, seguido pelo ambiente alterado $\left(25,42^{\circ} \mathrm{C}\right)$ e em transição $\left(25,17^{\circ} \mathrm{C}\right)$ (Figura 7$)$. Manhães e Francelino (2012) afirmam que os organismos se adaptam melhor em ambientes úmidos, sendo influenciados por fatores edafoclimáticos, como temperatura e umidade.

De acordo com Hess (1980), as matas atuam como reguladoras do clima. Dessa forma, as áreas de mata apresentam menor variabilidade em relação a temperatura. Ou seja, durante o dia a temperatura não se eleva muito, e durante a noite não baixa muito. Por isso, há uma tendência de formação de ventos leves da mata para os terrenos próximos durante o dia, e no sentido inverso à noite. Araujo (2002) em análise da temperatura da serapilheira em ambientes de mata e ambiente com cobertura vegetal moderada, verificou que no período da manhã, a temperatura obtida na camada de serapilheira é mais amena e à tarde esta já conserva-se mais aquecida, e atribui que a serapilheira desempenha um importante papel na manutenção do ritmo e/ou do balanço da temperatura no interior da floresta, uma vez que no período noturno a mesma torna-se mais aquecida, de modo a contribuir para que a mata não perca calor e consequentemente seja resfriada durante o referido período (HESS, 1980). Assim, pelo fato das leituras da temperatura do solo nas áreas experimentais terem sido realizadas nos primeiros horários do dia os valores levemente mais elevados na área conservada em relação aos demais ambientes foi atribuído a espessa camada de serapilheira que ultrapassa $0,10 \mathrm{~m}$ que é altura do termômetro. No entanto, a temperatura do solo abaixo da serapilheira é sempre mais reduzida nas áreas arborizadas em relação a área sem cobertura vegetal (BLEY JÚNIOR, 1999). Conforme se observou nas leituras das áreas de transição e área alterada. Dajoz (1978) afirma que uma fina camada de vegetação basta para reduzir consideravelmente o gradiente térmico existente acima e abaixo da superfície do solo nu, em decorrência do obstáculo oposto à radiação pelas plantas. O mesmo ocorre para a temperatura do ar que conforme indicam Martini et al. (2017) as diferentes tipologias de floresta urbana apresentam microclimas distintos, sendo que o remanescente florestal é o que proporciona valores menores de temperatura e maiores de umidade relativa. 
Figura 7 - Conteúdo de água do solo CAS (\%) e temperatura do solo $\left({ }^{\circ} \mathrm{C}\right)$, nos ambientes conservado, em transição e alterado.

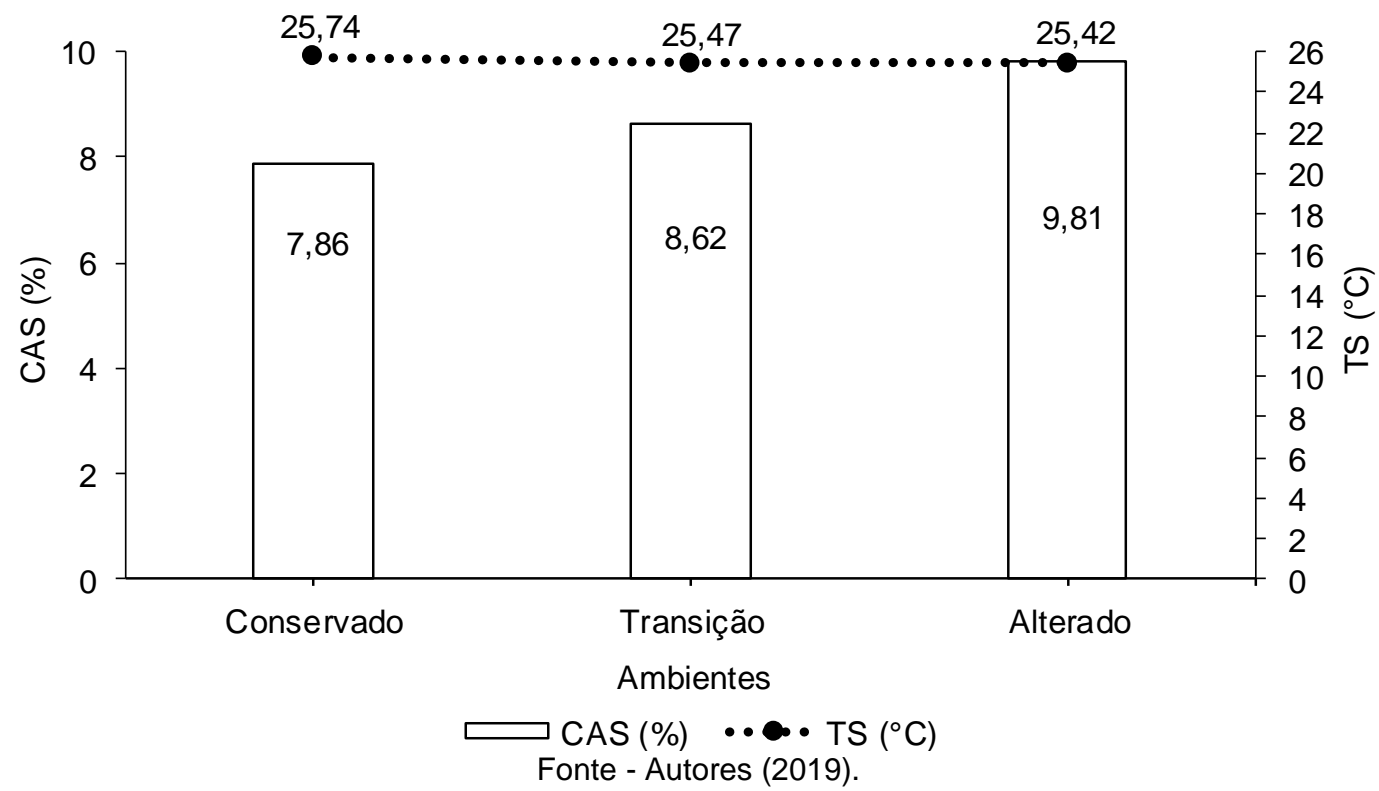

No ambiente em transição a cobertura vegetal é constituída por herbáceas e árvores com porte médio de $5 \mathrm{~m}$ e, a serapilheira é menos densa que a área conservada. Já no ambiente alterado o solo dispõe de espécies herbáceas e árvores com porte médio de $3 \mathrm{~m}$, com presença de espécies frutíferas como Ananas comosus (abacaxi) e no seu histórico de uso já foi plantado Manihot esculenta (macaxeira), apresentando pouco acúmulo de serapilheira (Figuras 2B e 2C).

Figura 8 - Precipitação pluvial (mm) bimestral de agosto de 2018 a junho de 2019.

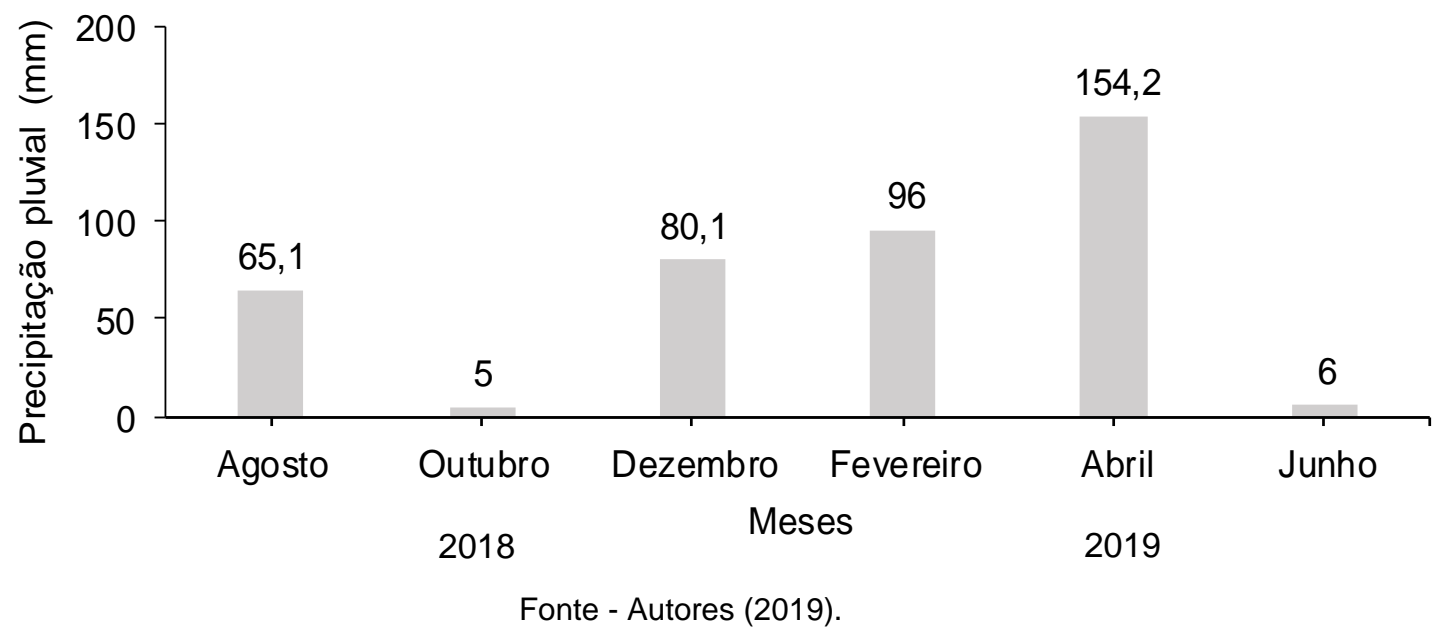

\section{ANALISE ESTATÍSTICA DA MACROFAUNA EDÁFICA}

De acordo com o teste de Tukey, a macrofauna apresentou diferença estatística entre as áreas. A abundância da macrofauna edáfica do local alterado diferiu estatisticamente dos ambientes conservado e em transição. E em relação à riqueza de grupos, todos diferiram entre si (Tabela 2). 
Tabela 2 - Médias da abundância e riqueza da macrofauna edáfica, nos ambientes conservado, em transição e alterado.

\begin{tabular}{lccc}
\hline \multicolumn{1}{c}{ Variáveis } & Conservado & Transição & Alterado \\
\hline Riqueza & $5,1 \mathrm{c}$ & $4,183 \mathrm{~b}$ & $3,433 \mathrm{a}$ \\
Abundância & $63,82 \mathrm{~b}$ & $32,92 \mathrm{a}$ & $33,87 \mathrm{a}$ \\
\hline
\end{tabular}

*Significativo ao nível de $5 \%$ de probabilidade.

Fonte - Autores (2019).

Ao analisar os dados dos testes de correlação (Tabela 3), pode-se verificar que todas as correlações tiveram baixos valores denotando que as correlações existentes para temperatura do solo e conteúdo de água do solo foram fracas e, para precipitação pluvial houve correlação moderada. No entanto, mesmo não havendo altas correlações, a temperatura do solo influenciou significativamente a abundância da macrofauna edáfica $\left(p=7,397 \mathrm{e}^{-06}\right)$. Foi verificado que o conteúdo de água do solo apresentou influência na riqueza $(p=0.03285)$ e a precipitação pluvial influenciou a abundância $(p=$ $\left.3,51 \mathrm{e}^{-09}\right)$ e a riqueza da macrofauna $\left(p=2,83 \mathrm{e}^{-05}\right.$ ) (Tabela 3 ). Santos et al. (2018), confirmam essa assertiva que em períodos mais chuvosos há maior abundância e riqueza dos organismos da macrofauna edáfica.

Tabela 3 - Estimativas de correlações de Pearson entre as variáveis abundância e riqueza da macrofauna edáfica relacionado com temperatura do solo (TS $\left.{ }^{\circ} \mathrm{C}\right)$, conteúdo de água no solo (CAS\%) e precipitação pluvial (PP $\mathrm{mm})$.

\begin{tabular}{lccc}
\hline \multicolumn{1}{c}{ Variáveis } & TS & CAS & PP \\
\hline Número de indivíduos & $0,327^{*}$ & $-0,119$ & $0,422^{*}$ \\
Número de grupos & 0,105 & $0,159^{*}$ & $0,307^{*}$
\end{tabular}

*Significativo ao nível de 5\% de probabilidade.

Fonte - Autores (2019).

Quando os valores de uma correlação se apresentam positivos, ou seja, quando uma variável aumenta, a outra variável também aumenta. E quando os valores são negativos, enquanto uma variável aumenta, a outra diminui. Cabe enfatizar que para que haja influência de uma variável sobre a outra, o valor da correlação tem que ser significativo. No entanto, mesmo o valor de uma correlação não sendo significativo, e nem tão baixo (por exemplo, acima de 0,4 ), pode-se dizer que há uma tendência de que uma variável interfira na outra, pois, como já mencionado anteriormente, para tal afirmação ser confirmada tem que haver significância.

Ao observar o conteúdo de água do solo com o número de indivíduos (abundância), e temperatura com número de grupos da macrofauna (riqueza de grupos) (Tabela 3), nota-se que os valores foram baixos (em torno de $r=0,1$ ) e sem significância, demonstrando que possivelmente, outras variáveis que não foram verificadas nesta pesquisa, é que estariam exercendo influência na macrofauna do solo.

\section{CONCLUSÕES}

- O ambiente conservado é o que apresenta maior riqueza e abundância, atribuído as condições favoráveis do ambiente em relação à variedade de alimento e abrigo encontrado na serapilheira, aliado aos fatores abióticos conteúdo de água do solo e temperatura; 
- Hymenoptera é o grupo dominante nos ambientes conservado, em transição e alterado, comprovado pelos baixos valores dos índices de Shannon e Pielou, por serem bem adaptados às condições antrópicas;

- O percentual de abundância do grupo Hymenoptera na área conservada não foi tão diferente e elevado quando comparado aos outros grupos que também apresentam características de grupos abundantes nessa mesma área. Isso denota que a área conservada está mais equilibrada que as demais;

- As variáveis edafoclimáticas influenciaram significativamente a macrofauna edáfica, sendo que a temperatura exerceu influência na abundância, o conteúdo de água do solo apresentou influência na riqueza e a precipitação pluvial influenciou ambas as variáveis;

- Recomenda-se a continuação de pesquisas sistemáticas da macrofauna edáfica a fim de indicar ações mitigadoras contra os impactos das intervenções antrópicas nos ecossistemas do Parque Municipal de Maceió. É preciso também realizar campanhas de conscientização da população do entorno para a conservação da mata, visando à manutenção do equilíbrio dos ecossistemas presentes no Parque, e a conservação de outras "ilhas verdes" na cidade. Os autores esperam que esta pesquisa sirva de referência para outros pesquisadores de áreas afins e que estes ampliem a pesquisa no Parque Municipal de Maceió e em outros parques de centros urbanos.

\section{REFERÊNCIAS}

ALMEIDA, M. A. X.; SOUTO, J. S.; ANDRADE, A. P. de. Sazonalidade da macrofauna edáfica do Curimataú da Paraíba, Brasil. Revista Ambiência, Guarapuava, v. 11, n. 2, p. 393-407, jan./abr. 2015. https://doi.org/10.5935/ambiencia.2015.02.09

ALVES, F. A. L. et al. Caracterização da macro e mesofauna edáfica sobre um fragmento remanescente de "mata atlântica" em Areia-PB. Revista Gaia Scientia, João Pessoa, n. 1, p. 384391, out./dez. 2014.

AQUINO, A.M. de; et al. Invertebrate soil macrofauna under different ground cover plants in the no-till system in the Cerrado. European Journal of Soil Biology, Amsterdam, v. 44, n. 2, p.191-197, mar./apr. 2008. https://doi.org/10.1016/j.ejsobi.2007.05.001

ARAUJO, K. D. Análise da vegetação e organismos edáficos em áreas de caatinga sob pastejo e aspectos socioeconômicos e ambientes de São João do Cariri - PB. 2010. 166 f. Tese (Doutorado em Recursos Naturais)-Centro de Tecnologia e Recursos Naturais, Universidade Federal de Campina Grande, Campina Grande, 2010.

ARAUJO, K. D. Diferenciação microclimática em paisagens com diferentes formas de cobertura vegetal: o caso do campus I da UFPB. 2002. 130 f. Monografia (Graduação em Geografia)-Centro de Ciências Exatas e da Natureza, Universidade Federal da Paraíba, João Pessoa, 2002.

ARCOVA, F. C. S.; CICCO, V. de; ROCHA, P. A. B. Precipitação efetiva e interceptação das chuvas por floresta de Mata Atlântica em uma microbacia experimental em Cunha - São Paulo. Revista Árvore, Viçosa, v. 27, n. 2, p. 257-262, mar./abr. 2003. https://doi.org/10.1590/S0100$\underline{67622003000200014}$

BARETTA, D. et al. Fauna edáfica e qualidade do solo. In: KLAUBERG-FILHO, O.; MAFRA, A. L.; GATIBONI, L. C. Tópicos em ciências do solo. Viçosa: Sociedade Brasileira de Ciência do Solo, 2011, p. 141-192.

BLEY JÚNIOR, C. Erosão solar: riscos para a agricultura nos trópicos. Ciência Hoje, Rio de Janeiro, v. 25 , n. 148 , p. 24-29. abr. 1999. 
BEGON, M.; HARPER, J. L.; TOWNSEND, C. R. Ecology: individuals, populations and communities. 3. ed. Oxford: Blackwell Science, 1996. 1068 p.

CABRERA, G. La macrofauna edáfica como indicador biológico del estado de conservación/perturbación del suelo. Resultados obtenidos em Cuba. Pastos Y Forrajes, Mantazas, v. 35, n. 4, p. 349-364. out./dez. 2012.

CIANCIARUSO, M.C. et al. Produção de serapilheira e decomposição do material foliar em um cerradão na Estação Ecológica de Jataí, município de Luiz Antônio, SP, Brasil. Acta Botanica Brasilica, São Paulo, v. 20, n. 1, p. 49-59. jan./mar. 2006. https://doi.org/10.1590/S0102$\underline{33062006000100006}$

DAJOZ, R. Ecologia geral. 3. ed. Petrópolis: Vozes, 1978. 472 p.

FACELLI, J. M.; PICKETT, S. T. A. Plant litter: its dynamics and effects on plant community structure. Botanical Review, Berlim, v. 57, n. 1, p. 1-32. jan. 1991. https://doi.org/10.1007/BF02858763

FILSER, J. et al. Soil fauna: key to new carbon models. Soil, Munique, v. 2, n. 4, p. 565-582. Nov. 2016. https://doi.org/10.5194/soil-2-565-2016

DANCEY, C. P.; REIDY, T. Estatística sem matemática para psicologia. 3. ed. Porto Alegre: Artmed, 2006. $608 \mathrm{p}$

GARCIA, D. V. B.; CATANOZI, G. Análise de macrofauna de solo em área de mata atlântica e de reflorestamento com Pinus sp. - zona sul de São Paulo. Revista Ibirapuera, São Paulo, v. 1, n. 2, p. 10-14, jul./dez. 2011.

HESS, A. A. Ecologia e produção agrícola. Florianópolis: ACARESC, 1980. 126 p.

INMET-INSTITUTO NACIONAL DE METEOROLOGIA. Normais Climatológicas do Brasil 19812010. Disponível em: www.inmet.gov.br. Acesso em: 25 mar. 2018.

LAVELLE, P. et al. Soil invertebrates and ecosystem services. European journal of soil biology, Rouen, v. 42, n. Supplement 1, p. 3-15, nov. 2006. https://doi.org/10.1016/j.ejsobi.2006.10.002

LIMA, B. M. Áreas de proteção permanente - APPs em Maceió: do ideário conservacionista aos usos socioambientais das zonas de interesses ambiental e paisagístico. 2009. $140 \mathrm{f}$. Dissertação (Mestrado em Arquitetura e Urbanismo)-Faculdade de Arquitetura e Urbanismo, Universidade Federal de Alagoas, Maceió, 2009.

MACHADO, D. L. et al. Fauna edáfica na dinâmica sucessional da mata atlântica em floresta estacional semidesidual na bacia do rio Paraíba do Sul - RJ. Ciência Florestal, Santa Maria, v. 25, n. 1, p. 91-106, jan./mar. 2015. https://doi.org/10.5902/1980509817466

MANHÃES, C. M. C.; FRANCELINO, F. M. A. Estudo da inter-relação da qualidade do solo e da serapilheira com a fauna edáfica utilizando análise multivariada. Nucleus, Ituverava, v. 9, n. 2, p. 21 32, out. 2012. https://doi.org/10.3738/1982.2278.701

MARQUES, G. D. V.; DEL-CLARO, K. Sazonalidade, abundância e biomassa de insetos de solo em uma reserva de Cerrado. Revista Brasileira de Zoociências, Juiz de Fora, v. 12, n. 2, p. 141-150, jun. 2010.

MATEUS, F. A. et al. Estoque e capacidade de retenção hídrica da serrapilheira acumulada na restauração florestal de áreas perturbadas na mata atlântica. Floresta e Ambiente, Pinheiral, v. 20 n. 3, p. 336-343, ago. 2013. https://doi.org/10.4322/floram.2013.024

MARTINI, A. et al. Análise microclimática das diferentes tipologias de floresta urbana de Curitiba. 
Floresta, Curitiba, v. 47, n. 2, p. 137-144, abr./jun. 2017. https://doi.org/10.5380/rf.v47i2.49518

MELO, F. V. de et al. A importância da meso e macrofauna do solo na fertilidade e como bioindicadores. Boletim Informativo da Sociedade Brasileira de Ciência do Solo, Viçosa, v. 34, n. 1, p. 39-43, jan./abr. 2009.

MERLIM, A. de O. Macrofauna edáfica em ecossistemas preservados e degradados de araucária no Parque Estadual de Campos de Jordão, SP. 2005. 103 f. Dissertação (Mestrado em Ecologia de Agroecossistemas)-Escola Superior de Agricultura Luiz de Queiroz, Universidade de São Paulo, Piracicaba, 2005.

MIRANDA, J. C. Intercepção das chuvas pela vegetação florestal e serrapilheira nas encostas do Maciço da Tijuca: Parque Nacional da Tijuca, RJ. 1992. 100 f. Dissertação (Mestrado em Geografia)-Instituto de Geografia, Universidade Federal do Rio de Janeiro, Rio de Janeiro, 1992.

MITTERMEIER, R. A. et al. Hotspots revisited. Earth's biologically richest and most endangered Terrestrial ecoregions. 2. ed. Mexico City: CEMEX, 2005. $392 \mathrm{p}$.

MORAES, R. M. Ciclagem de nutrientes na floresta do PEFI: produção e decomposição de serapilheira. In: BICUDO, E. de M.; FORTI, M. C.; BICUDO, D de C. (Orgs). Parque Estadual das Fontes do Ipiranga: uma Reserva Biológica na cidade de São Paulo. 1. ed. São Paulo: Secretaria do Meio Ambiente do Estado de São Paulo, 2002. p. 133-142.

MYERS, N. et al. Biodiversity hotspots for conservation priorities. Nature, Oxford, v. 403. n. 6772, p. 853-858, fev. 2000. https://doi.org/10.1038/35002501

OLIVEIRA, R. R. Produção e decomposição de serapilheira no Parque Nacional da Tijuca, RJ. 1987.107 f. Dissertação (Mestrado em Geografia)-Instituto de Geociências, Universidade Federal do Rio de Janeiro, Rio de Janeiro,1987.

PATUCCI, N. N. et al. Inventário de fauna edáfica como instrumento na avaliação de qualidade e biodiversidade de solos urbanos: estudo de caso do parque CienTec. Boletim Paulista de Geografia, São Paulo, v. 96, n. 1, p. 66-90. jan./jun. 2017. https://doi.org/10.11606/rdg.v36i0.149144

PATUCCI, N. N. Bioindicadores edáficos de fragmentos florestais urbanos da Cidade de São Paulo (SP). Revista do Departamento de Geografia, São Paulo, v. 36, n. 2, p. 77-90, jun./dez. 2018.

$\mathrm{PECH}, \mathrm{T}$. M. et al. Macrofauna edáfica em três sistemas agroflorestais no município de Curitibanos, SC. In: XII SIMPÓSIO FLORESTAL CATARINENSE 12, 2016, Lages. Anais... Lages: CAV/UDESC, 2016, p. 129-134.

PEEL, M. C.; FINLAYSON, B. L.; MCMAHON, T. A. Updated world map of the Koppen-Geiger climate classification. Hydrology and Earth System Sciences, Germany, v. 11, n. 5, p. 1633-1644. oct. 2007. https://doi.org/10.5194/hess-11-1633-2007

PENEIREIRO, F. M. Sistemas agroflorestais dirigidos pela sucessão natural: um estudo de caso. 1999. 138 f. Dissertação (Mestrado em Ciências)-Escola Superior de Agricultura Luiz de Queiroz, Universidade de São Paulo, Piracicaba, 1999.

PIELOU, E. C. Mathematical ecology. 2. ed. New York: John Wiley \& Sons, 1977. 385 p.

PIMENTEL, A. K, S. et al. Observações das ações antrópicas e seus impactos ambientais negativos no parque municipal de Maceió. $V$ CONGRESSO NORTE-NORDESTE DE PESQUISA E INOVAÇÃO, 5, 2010, Maceió Anais... Maceió: SETEC, 2010. p. 1-8.

POMPEO, P. N. et al. Fauna e sua relação com atributos edáficos em Lages, Santa Catarina - Brasil. Revista Scientia Agraria, Curitiba, v. 17, n. 1, p. 42-51, jan./mar. 2016. https://doi.org/10.5380/rsa.v17i1.46535 
R CORE TEAM. R: a language and environment for statistical computing. Vienna: R Foundation for Statistical Computing, 2019. Disponível em: https://www.R-project.org. Acesso em: 29 ago 2020.

RUIZ, N.; LAVELLE, P.; JIMENEZ, J. Soil macrofauna field manual: Technical level. Rome: Food and Agriculture Organization of the United Nations, 2008. $101 \mathrm{p}$.

SANTOS, G. R. dos; ARAUJO, K. D.; SILVA, F. G. Macrofauna edáfica na Estação Ecológica Curral do Meio, Caatinga Alagoana. Revista de Geociências do Nordeste, Caicó, v. 4, n. 2, p. 1-21, jul. 2018.

SATO, A. M. Respostas geo-hidroecológicas relacionadas à substituição de pastagens por plantações de eucalipto no Médio Vale do Rio Paraíba do Sul: a interface biota-solo-água. 2008. 160 f. Dissertação (Mestrado em Geografia)-Instituto de Geociências, Universidade Federal do Rio de Janeiro, Rio de Janeiro, 2008.

SILVA, A. C. F. da. et al. Macrofauna edáfica em três diferentes usos do solo. Enciclopédia Biosfera: Centro Científico Conhecer, Goiânia, v. 10, n. 18, p. 2131-2137, jul. 2014.

SILVA, W. P. da. Análise do Parque Municipal como atrativo turístico de Maceió-AL. 2017. 56 f. Trabalho de Conclusão de Curso (Graduação em Geografia)-Instituto de Geografia, Desenvolvimento e Meio Ambiente, Universidade Federal de Alagoas, Maceió, 2017.

SILVA, V. M. F. da. Efeito das ações antrópicas na qualidade da água da bacia do Riacho do Silva, em Maceió-AL. 2011. 178 f. Dissertação (Mestrado em Recursos Hídricos e Saneamento, Centro de Tecnologia, Universidade Federal de Alagoas, Maceió, 2011.

SOUZA, M. H. et al. Macrofauna do solo. Enciclopédia Biosfera: Centro Científico Conhecer, Goiania, v. 11, n. 22, p. 115-131, jul./dez. 2015.

SWIFT, M. J.; HEAL, O. W.; ANDERSON, J. M. Decomposition in terrestrial ecosystems: studies in ecology. v. 5. Oxford: Blackwell Scientific, 1979. 238 p.

TEDESCO, J. M.; VOLKWEISS, S. J.; BOHNEN, H. Análises do solo, plantas e outros materiais. 1. ed. Porto Alegre: Universidade Federal do Rio Grande do Sul, 1995. 188 p. (Boletim técnico).

VALLEJO, L. R. A influência do litter na distribuição das águas pluviais. 1982. 123 f. Dissertação (Mestrado em Geografia)-Instituto de Geografia, Universidade Federal do Rio de Janeiro, Rio de Janeiro, 1982.

Recebido em: 04/06/2020

Aceito para publicação em: 03/09/2020 